This item was submitted to Loughborough's Research Repository by the author.

Items in Figshare are protected by copyright, with all rights reserved, unless otherwise indicated.

\title{
Improved eigenvalue bounds for Schrödinger operators with slowly decaying potentials
}

PLEASE CITE THE PUBLISHED VERSION

https://doi.org/10.1007/s00220-019-03635-w

\section{PUBLISHER}

Springer (part of Springer Nature)

\section{VERSION}

AM (Accepted Manuscript)

\section{PUBLISHER STATEMENT}

This is a post-peer-review, pre-copyedit version of an article published in Communications in Mathematical Physics. The final authenticated version is available online at: https://doi.org/10.1007/s00220-019-03635-w.

\section{LICENCE}

CC BY-NC-ND 4.0

\section{REPOSITORY RECORD}

Cuenin, Jean-Claude. 2019. "Improved Eigenvalue Bounds for Schrödinger Operators with Slowly Decaying Potentials”. Loughborough University. https://hdl.handle.net/2134/11864982.v1. 


\title{
IMPROVED EIGENVALUE BOUNDS FOR SCHRÖDINGER OPERATORS WITH SLOWLY DECAYING POTENTIALS
}

\author{
JEAN-CLAUDE CUENIN
}

\begin{abstract}
We extend a result of Davies and Nath 5 on the location of eigenvalues of Schrödinger operators with slowly decaying complex-valued potentials to higher dimensions. In this context, we also discuss various examples related to the Laptev-Safronov conjecture [21].
\end{abstract}

\section{INTRODUCTION AND MAIN RESULT}

Eigenvalue estimates for Schrödinger operators $-\Delta+V$ on $L^{2}\left(\mathbb{R}^{d}\right)$ with complexvalued potentials $V \in L^{q}\left(\mathbb{R}^{d}\right)$ have been intensively studied over the past two decades by many authors, starting with the observation of Abramov, Aslanyan and Davies [1] that in one dimension the bound

$$
|z|^{\frac{1}{2}} \leq \frac{1}{2} \int_{\mathbb{R}}|V(x)| \mathrm{d} x
$$

holds for any eigenvalue $z \in \mathbb{C} \backslash[0, \infty)$ of $-\Delta+V$. This scale-invariant bound has the same form (up to replacing $|V(x)|$ by $V(x)_{-}$) as the endpoint Lieb-Thirring inequality in $d=1$ ([23, 18]) for a potential with a single eigenvalue. Higherdimensional versions of (1.1) were proved by Frank [11, 12] and Frank-Simon [16. A maybe less well-known bound in the one-dimensional case, due to Davies and Nath [5], improves (1.1) to

$$
|z|^{\frac{1}{2}} \leq \frac{1}{2} \sup _{y \in \mathbb{R}} \int_{\mathbb{R}}|V(x)| \exp (-\operatorname{Im} \sqrt{z}|x-y|) \mathrm{d} x,
$$

which is valid even for slowly decaying potentials, i.e. $V \notin L^{1}(\mathbb{R})$. The aim of this note is to prove the following higher-dimensional analogue of (1.2).

Theorem 1.1. Let $d \geq 2, q \in[\max (d / 2,1+),(d+1) / 2]$ and $V \in L_{\mathrm{loc}}^{q}\left(\mathbb{R}^{d}\right)$. Then there exists $C_{d, q}>0$ such that any eigenvalue $z \in \mathbb{C}$ of $-\Delta+V$ satisfies

$$
|z|^{q-\frac{d}{2}} \leq C_{d, q} \sup _{y \in \mathbb{R}^{d}} \int_{\mathbb{R}^{d}}|V(x)|^{q} \exp (-\operatorname{Im} \sqrt{z}|x-y|) \mathrm{d} x .
$$

Here, $1+$ denotes an arbitrary number $>1$ (this is only relevant if $d=2$ ) and the branch of the square root is chosen such that $\operatorname{Im} \sqrt{z}>0$. In the "short-range" case $V \in L^{q}\left(\mathbb{R}^{d}\right), q \leq(d+1) / 2$, Theorem 1.1 recovers the results of [11, simply by neglecting the exponential. In the "long-range" case $q>(d+1) / 2$ an application of Hölder's inequality yields bounds that are close to those of [12]; see Corollary 3.1 and the subsequent remark for details. We also mention the recent result of Lee and Seo 22] where the $L^{q}$ norm is replaced by the Kerman-Saywer norm. 
An interesting consequence of our bound (1.3) that cannot be deduced from those works is that, for a long-range potential $V$, we have the "local bound"

$$
|z|^{\frac{1}{2}} \leq C_{d} \sup _{y \in \mathbb{R}^{d}} \int_{\left(B\left(y, \frac{M}{\operatorname{Im} \sqrt{z}}\right)\right)}|V(x)|^{\frac{d+1}{2}} \mathrm{~d} x,
$$

where $M$ is some large number depending on $V$ and $z$ (see Corollary 3.3). If more is known about the potential than just an $L^{q}$ norm, then (1.4) can yield considerably sharper bounds than those previously known in the literature. For example, we show that if $V$ is of "Ionescu-Jerison" type in the sense discussed in 16, then the imaginary part of $z$ must decay exponentially fast as the "size" of $V$ tends to zero (see Example 5.2). This observation may give a hint whether such a potential is a good candidate to disprove the open part of the so-called Laptev-Safronov conjecture [21, which stipulates that

$$
\sup _{V \in L^{q}\left(\mathbb{R}^{d}\right)} \sup _{z \in \sigma(-\Delta+V) \backslash \mathbb{R}_{+}} \frac{|z|^{q-\frac{d}{2}}}{\|V\|_{q}^{q}}<\infty \quad \text { for all } \quad q \in[d / 2, d] .
$$

For the range $q \in[d / 2,(d+1) / 2]$ the conjecture was proven by Frank [11. The question whether (1.5) is true for $q \in((d+1) / 2, d]$ is still open. The lower bound for $q$ in (1.5) is obvious and already appears for real-valued potentials. The conjectured upper bound $q \leq d$ was based on the observation that there are examples of potentials, due to Wigner and Von Neumann, that decay like $1 /|x|$ at infinity and give rise to embedded eigenvalues. For these potentials $q=d$ would be borderline in terms of integrability. However, there are examples of embedded eigenvalues, due to Ionescu and Jerison [19], for non-radial potentials that are in $L^{q}\left(\mathbb{R}^{d}\right)$ for any $q>(d+1) / 2$; see also [16 for a simplified version of the Ionescu-Jerison example and 4 for additional examples as well as an explanation of the connection to the Knapp example in harmonic analysis. Thus, the expectation is that the conjecture is false in the range $q \in((d+1) / 2, d]$. That (1.5) cannot hold for $q>d$, as conjectured by Laptev and Safronov, was proved by Bögli [2].

Finally we should also mention that there are versions of (1.1) concerning sums of eigenvalues (e.g. 13, 6, 7, 15, 14), but these will not be discussed here. Several works also deal with a class of potentials outside the $L^{q}$-scale (e.g. [10, 9]), Schrödinger operators with inverse square potentials 24], Schrödinger operators on conical manifolds with non-trapping metrics [17, fractional Schrödinger and Dirac operators [3], to name just a few.

\section{Proof of Theorem 1.1}

To ease notation we define

$$
F_{V}^{q}(s):=\left(\sup _{y \in \mathbb{R}^{d}} \int_{\mathbb{R}^{d}}|V(x)|^{q} \exp (-s|x-y|) \mathrm{d} x\right)^{\frac{1}{q}} .
$$

The first step is to reduce the proof of Theorem 1.1 to the Birman-Schwinger bound

$$
\left\||V|^{\frac{1}{2}}(-\Delta-z)^{-1}|V|^{\frac{1}{2}}\right\| \leq C_{d, q}|z|^{\frac{d}{2 q}-1} F_{V}^{q}(\operatorname{Im} \sqrt{z}) .
$$

We first prove that it is sufficient to establish (2.1) for $|z|=1$, then reduce the proof to a pointwise bound.

Lemma 2.1 (Scaling). If (2.1) holds for $|z|=1$, then it holds for all $z \in \mathbb{C}$. 
Proof. One easily checks that

$$
\left\||V|^{\frac{1}{2}}(-\Delta-z)^{-1}|V|^{\frac{1}{2}}\right\|=|z|^{-1}\left\||V(\cdot / \sqrt{|z|})|^{\frac{1}{2}}(-\Delta-z /|z|)^{-1}|V(\cdot / \sqrt{|z|})|^{\frac{1}{2}}\right\| .
$$

Hence, if (2.1) held for $|z|=1$ this would imply that

$$
\left\||V|^{\frac{1}{2}}(-\Delta-z)^{-1}|V|^{\frac{1}{2}}\right\| \leq C_{d, q}|z|^{-1} F_{V(\cdot / \sqrt{|z|})}^{q}(\operatorname{Im} \sqrt{z /|z|}) .
$$

Since

$$
F_{V(\cdot / \sqrt{|z|})}^{q}(s)=|z|^{\frac{d}{2 q}} F_{V}^{q}(s \sqrt{|z|}),
$$

the claim follows.

Lemma 2.2 (Pointwise bounds). Let $z, \zeta \in \mathbb{C}$, with $|z|=1, \operatorname{Im} z \neq 0$ and $\operatorname{Re} \zeta \in$ $[d / 2,(d+1) / 2]$. Then the kernel $K_{z, \zeta}$ of $(-\Delta-z)^{-\zeta}$ satisfies the bound

$$
\left|K_{z, \zeta}(x-y)\right| \leq C \mathrm{e}^{c|\operatorname{Im} \zeta|} \mathrm{e}^{-\operatorname{Im} \sqrt{z}|x-y|}|x-y|^{-\frac{d+1}{2}+\operatorname{Re} \zeta} .
$$

Proof. This follows from the explicit formula for the kernel $K_{z, \zeta}$ and standard Bessel function estimates, see e.g. (2.21)-(2.27) in [20] or the proof of (2.5) in the appendix of [22] (where the estimate is in fact proved for the larger range $\operatorname{Re} \zeta \in[(d-1) / 2,(d+1) / 2]$, but this will not be needed here). In both references the (second) exponential factor in (2.2) is simply estimated by one.

We need the following simple version of Schur's test. The proof follows from a routine application of the Cauchy-Schwarz inequality and is omitted.

Lemma 2.3 (Schur's test). Let $\rho: \mathbb{R}^{d} \times \mathbb{R}^{d} \rightarrow(0, \infty)$. Suppose that $T$ is an operator on $L^{2}\left(\mathbb{R}^{d}\right)$ with locally integrable kernel $K$. Then

$$
\|T\|_{L^{2} \rightarrow L^{2}} \leq\left(\sup _{x \in \mathbb{R}^{d}} \int_{\mathbb{R}^{d}}|K(x, y)| \rho(x, y)^{-1} \mathrm{~d} y\right)^{\frac{1}{2}}\left(\sup _{y \in \mathbb{R}^{d}} \int_{\mathbb{R}^{d}}|K(x, y)| \rho(x, y) \mathrm{d} x\right)^{\frac{1}{2}} .
$$

Proof of Theorem 1.1. We will apply Stein's complex interpolation theorem (see e.g. 25] for a textbook reference) to the analytic family of operators

$$
T_{\zeta}=|V|^{\frac{\zeta}{2}}(-\Delta-z)^{-\zeta}|V|^{\frac{\zeta}{2}}
$$

where $|z|=1$ and $0 \leq \operatorname{Re} \zeta \leq(d+1) / 2$ (see also [15, 3, 12, 22] where the same family is considered). It suffices to prove the bounds

$$
\begin{aligned}
\left\|T_{\zeta}\right\|_{L^{2} \rightarrow L^{2}} \leq C \mathrm{e}^{c|\operatorname{Im} \zeta|} & \text { for } \operatorname{Re} \zeta=0, \\
\left\|T_{\zeta}\right\|_{L^{2} \rightarrow L^{2}} \leq C \mathrm{e}^{c|\operatorname{Im} \zeta|} F_{V}^{q}(\operatorname{Im} \sqrt{z}) & \text { for } \operatorname{Re} \zeta=q
\end{aligned}
$$

The first bound immediately follows from Plancherel's theorem (see e.g. the proof of (2.3) in 22]). The second bound follows from (2.2) and Schur's test with

$$
\rho(x, y)=\frac{|V(x)|^{\frac{q}{2}}}{|V(y)|^{\frac{q}{2}}} .
$$

To be precise, we first truncate $|V|$ from above and below, so that $\rho$ and $\rho^{-1}$ are bounded. The truncation can be removed at the end. 


\section{Consequences of Theorem 1.1}

Corollary 3.1. Let $d \geq 2$ and $V \in L^{q}\left(\mathbb{R}^{d}\right)$ for some $q \geq(d+1) / 2$. Then there exists $C_{d, q}>0$ such that any eigenvalue $z \in \mathbb{C}$ of $-\Delta+V$ satisfies

$$
|z|^{\frac{1}{d+1}}(\operatorname{Im} \sqrt{z})^{d\left(\frac{2}{d+1}-\frac{1}{q}\right)} \leq C_{d, q}\|V\|_{q}
$$

Proof. This follows from (1.3) with $q=(d+1) / 2$ by Hölder's inequality.

Remark 3.2. Since $\operatorname{Im} \sqrt{z} \gtrsim \operatorname{dist}\left(z, \mathbb{R}_{+}\right) / \sqrt{|z|}$ it immediately follows from (3.1) that

$$
|z|^{\frac{1}{d+1}-\frac{d}{2}\left(\frac{2}{d+1}-\frac{1}{q}\right)} \operatorname{dist}\left(z, \mathbb{R}_{+}\right)^{d\left(\frac{2}{d+1}-\frac{1}{q}\right)} \leq C_{d, q}\|V\|_{q} .
$$

This estimate is not so good for large $q$; for example, for $q=\infty$ the trivial bound

$$
\operatorname{dist}\left(z, \mathbb{R}_{+}\right) \leq\|V\|_{\infty}
$$

easily beats (3.1) since $\operatorname{dist}\left(z, \mathbb{R}_{+}\right) \leq|z|$. In terms of the Birman-Schwinger operator the inequality leading to (3.3) is of course

$$
\left\||V|^{\frac{1}{2}}(-\Delta-z)^{-1}|V|^{\frac{1}{2}}\right\| \leq \operatorname{dist}\left(z, \mathbb{R}_{+}\right)^{-1}\|V\|_{\infty} .
$$

Since the right hand side of (2.1) is clearly bounded by $|z|^{\frac{1}{d+1}}\|V\|_{d+1}$, complex interpolation between (2.1) and (3.4) yields

$$
|z|^{\frac{1}{2 q}} \operatorname{dist}\left(z, \mathbb{R}_{+}\right)^{1-\frac{d+1}{2 q}} \leq C_{d, q}\|V\|_{q}
$$

for $q \geq(d+1) / 2$. This bound was proved by Frank [12], and we refer to this paper for the details of the complex interpolation.

Corollary 3.3. Let $d \geq 2$ and $V \in L^{q}\left(\mathbb{R}^{d}\right)$ for some $q>(d+1) / 2$. If $z \in \mathbb{C}$ is an eigenvalue of $-\Delta+V$, then there exists $C_{d}^{\prime}>0$ such that for any $M \geq 0$ satisfying

$$
M \geq(d+1) \ln \|V\|_{q}-2 d \beta_{q}^{-1} \ln \left(\beta_{q} \operatorname{Im} \sqrt{z}\right)-\ln |z|+(d+1) \ln \left(2 C_{d}^{\prime}\right),
$$

where $\beta_{q}^{-1}=1-(d+1) /(2 q)$, the bound

$$
|z|^{\frac{1}{d+1}} \leq 2 C_{d}^{\prime} \sup _{y \in \mathbb{R}^{d}}\|V\|_{L^{\frac{d+1}{2}}}\left(B\left(y, \frac{M}{\operatorname{Im} \sqrt{z}}\right)\right)
$$

holds.

Proof. We split the integral in (1.3) (again with $q=(d+1) / 2$ ) into a region $|x-y| \leq M / \operatorname{Im} \sqrt{z}$ and its complement. Estimating the exponential factor by 1 in the first region and using Hölder in the second yields

$$
|z|^{\frac{1}{d+1}} \leq C_{d}^{\prime}\left(\sup _{y \in \mathbb{R}^{d}}\|V\|_{L^{\frac{d+1}{2}}\left(B\left(y, \frac{M}{\ln \sqrt{z}}\right)\right)}+\mathrm{e}^{-\frac{M}{d+1}}\left(\beta_{q} \operatorname{Im} \sqrt{z}\right)^{-d\left(\frac{2}{d+1}-\frac{1}{q}\right)}\|V\|_{q}\right)
$$

for some constant $C_{d}^{\prime}>0$ that is a multiple of $C_{d, \frac{d+1}{2}}$ in (1.3). By the choice of $M$ in (3.6) the second term is at most half the size of the left hand side and can thus be absorbed. 


\section{A ShaRP BOUND For QUASIMODES}

In this section we prove a statement that is slightly stronger than that in [12]. Example 5.6 below shows that this stronger version is sharp. Since (1.3) is scaleinvariant, we may assume that $|z|=1$ in the following. We consider the following generalized eigenvalue or quasimode equation,

$$
\left(-\Delta+V_{n}-z_{n}\right) \psi_{n}=g_{n},
$$

where $g_{n}$ is a suitably small error, made precise in (4.2) below.

Proposition 4.1. Let $\epsilon_{n}$ be a sequence of positive numbers tending to zero, and let $z_{n}$ be a sequence of complex numbers with $\left|z_{n}\right| \approx 1$ and $\operatorname{Im} z_{n}=\epsilon_{n}$. Assume that there exist functions $\psi_{n} \in H^{2}\left(\mathbb{R}^{d}\right), g_{n} \in L^{2}\left(\mathbb{R}^{d}\right)$ and $V_{n} \in L^{q}\left(\mathbb{R}^{d}\right)$, for some $q>(d+1) / 2$, such that (4.1) holds. Then there exists $\delta>0$ such that if

$$
\limsup _{n \rightarrow \infty} \epsilon_{n}^{\frac{d+1}{4 q}-1}\left\|V_{n}\right\|_{q}^{\frac{1}{2}} \frac{\left\|g_{n}\right\|_{2}}{\left\|V_{n}^{\frac{1}{2}} \psi_{n}\right\|_{2}} \leq \delta
$$

then the bound

$$
\epsilon_{n}^{1-\frac{d+1}{2 q}} \leq C_{d, q}\left\|V_{n}\right\|_{q}
$$

holds for sufficiently large $n$.

Remark 4.2. If $g_{n}=0$, then condition (4.2) is void, and we recover the eigenvalue bound (3.5) for fixed $n$.

For the proof of Proposition 4.1 we will need the following consequence of the Stein-Tomas theorem.

Lemma 4.3. Let $n \geq 2$. Then, for $\lambda^{-1} \leq \epsilon \leq 1$, we have

$$
\left\|\left(-\Delta-(\lambda+\mathrm{i} \epsilon)^{2}\right)^{-1}\right\|_{L^{2} \rightarrow L^{p_{c}}} \lesssim \epsilon^{-\frac{1}{2}} \lambda^{\frac{1}{p_{c}}-1} \quad\left(p_{c}=2(d+1) /(d-1)\right) .
$$

Proof. The proof is essentially contained in 20. For completeness, we provide a full proof here (communicated to the author by C.D. Sogge). By duality, and due to the assumptions on $\epsilon, \lambda$, it is sufficient to prove that

$$
\left\|\int \frac{\mathrm{e}^{\mathrm{i} x \cdot \xi} \widehat{f}(\xi)}{|\xi|^{2}-\lambda^{2}-\mathrm{i} \epsilon \lambda} \mathrm{d} \xi\right\|_{2} \lesssim \epsilon^{-\frac{1}{2}} \lambda^{\frac{1}{p_{c}}-1}\|f\|_{p_{c}^{\prime}} .
$$

The Stein-Tomas theorem asserts that

$$
\left(\int_{S^{d-1}}|\widehat{h}(\omega)|^{2} \mathrm{~d} \omega\right)^{\frac{1}{2}} \lesssim\|h\|_{p_{c}^{\prime}}
$$

By scaling, (4.6) is equivalent to

$$
\left(\int_{S^{d-1}}|\widehat{h}(r \omega)|^{2} r^{d-1} \mathrm{~d} \omega\right)^{\frac{1}{2}} \lesssim r^{\frac{1}{p_{c}}}\|h\|_{p_{c}^{\prime}}
$$

\footnotetext{
${ }^{1}$ Note that $\left\|V_{n}^{\frac{1}{2}} \psi_{n}\right\|_{2}$ is finite in view of Sobolev embedding.
} 
Using polar coordinates, Plancherel's theorem and (4.7), we get, since $p_{c}>2$,

$$
\begin{aligned}
\left\|\int \frac{\mathrm{e}^{\mathrm{i} x \cdot \xi} \widehat{f}(\xi)}{|\xi|^{2}-\lambda^{2}-\mathrm{i} \epsilon \lambda} \mathrm{d} \xi\right\|_{2}^{2} & \lesssim \lambda^{-2} \int_{0}^{\infty} \int_{S^{d-1}}|\widehat{f}(r \omega)|^{2} r^{d-1} \mathrm{~d} \omega \frac{\mathrm{d} r}{(r-\lambda)^{2}+\epsilon^{2}} \\
& \lesssim \lambda^{-2}\left(\int_{0}^{\infty} \frac{r^{\frac{2}{p_{c}}}}{(r-\lambda)^{2}+\epsilon^{2}} \mathrm{~d} r\right)\|f\|_{p_{c}^{\prime}}^{2} \\
& \lesssim \epsilon^{-1} \lambda^{\frac{2}{p_{c}}-2}\|f\|_{p_{c}^{\prime}}^{2} .
\end{aligned}
$$

Corollary 4.4. For $q>(d+1) / 2,|z| \approx 1,|\operatorname{Im} z|=\epsilon \ll 1$, we have

$$
\left\|f(-\Delta-z)^{-1} g\right\| \lesssim \epsilon^{-1+\frac{d+1}{4 q}}\|g\|_{2}\|f\|_{2 q} .
$$

Proof. Without loss of generality we may assume that $f, g>0$. We apply Stein's interpolation theorem to the analytic family

$$
T_{\zeta}=f^{\zeta}(-\Delta-z)^{-1}, \quad 0 \leq \operatorname{Re} \zeta \leq \frac{2 q}{d+1} .
$$

It is sufficient to prove the two estimates

$$
\begin{aligned}
\left\|T_{\zeta}\right\| \leq \epsilon^{-1}, & \operatorname{Re} \zeta=0, \\
\left\|T_{\zeta}\right\| \lesssim \epsilon^{-\frac{1}{2}}\|f\|_{q}^{\frac{q}{d+1}}, & \operatorname{Re} \zeta=\frac{2 q}{d+1} .
\end{aligned}
$$

The first is just the trivial bound (3.4). The second follows from (4.4) and Hölder.

Proof of Proposition 4.1. From the quasimode equation (4.1) it follows that

$$
\left|V_{n}\right|^{\frac{1}{2}} \psi_{n}=\left|V_{n}\right|^{\frac{1}{2}}\left(-\Delta-z_{n}\right)^{-1} g_{n}-\left|V_{n}\right|^{\frac{1}{2}}\left(-\Delta-z_{n}\right)^{-1} V_{n}^{\frac{1}{2}}\left(\left|V_{n}^{\frac{1}{2}}\right| \psi_{n}\right) .
$$

By (4.8) this implies that

$$
1 \lesssim\left\|V_{n}^{\frac{1}{2}}\left(-\Delta-z_{n}\right)^{-1} V_{n}^{\frac{1}{2}}\right\|+\epsilon^{-1+\frac{d+1}{4 q}}\left\|V_{n}\right\|_{q}^{\frac{1}{2}} \frac{\left\|g_{n}\right\|_{2}}{\left\|V_{n}^{\frac{1}{2}} \psi_{n}\right\|_{2}} .
$$

By assumption (4.2) we can absorb the second term. Then the usual BirmanSchwinger argument applies and yields (4.3), in parallel to the poof of (3.5).

\section{EXAMPLES}

Example 5.1. Fix $q>(d+1) / 2, \mu \in(0,1]$, and consider the ball

$$
\mathcal{B}_{q, \mu}:=\left\{V \in L^{q}\left(\mathbb{R}^{d}\right):\|V\|_{q} \leq \mu\right\} .
$$

Also fix a small number $\delta>0$, and consider the rectangle

$$
\Omega_{\delta}:=\{z \in \mathbb{C}: 1 \leq \operatorname{Re} z \leq 2,0<\operatorname{Im} z \leq \delta\}
$$

in the upper half plane (the horizontal position is not so important, only boundedness of $\operatorname{Re} z$ from above and below, i.e. away from zero, is needed). Note that $\operatorname{Im} \sqrt{z}=(\operatorname{Im} z) / 2+\mathcal{O}\left(\delta^{2}\right)$ for $z \in \Omega_{\delta}$, as $\delta$ tends to zero. Assume that $z \in \Omega_{\delta}$ is an eigenvalue of $-\Delta+V$, where $V \in \mathcal{B}_{q, \mu}$. Let $\delta$ be so small that the last term in (3.6) 
is bounded from above by $-d \beta_{q}^{-1} \ln \left(\beta_{q} \delta\right)$. Dropping negative terms in (3.6) we may choose $M:=-3 d \beta_{q}^{-1} \ln \left(\beta_{q} \operatorname{Im} z\right)$. Corollary 3.3 then implies the lower bound

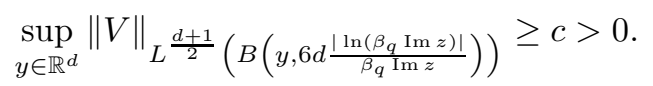

In other words, a potential $V \in \mathcal{B}_{q, \mu}$ giving rise to an eigenvalue $z \in \Omega_{\delta}$ must have positive $L^{\frac{d+1}{2}}$ mass over some ball of radius $|\ln \operatorname{Im} z| / \operatorname{Im} z$.

Example 5.2. This is a continuation of the previous example. We consider a family of potentials $V_{n}$, depending on a large parameter $n$ and satisfying the upper bound

$$
\left|V_{n}(x)\right| \lesssim\left(n+\left|x_{1}\right|+\left|x^{\prime}\right|^{2}\right)^{-1} .
$$

Such potentials naturally appear in examples of absence of embedded eigenvalues, see Ionescu-Jerison [19], Frank-Simon [16] and the author [4]. Denote

$$
U_{n, \kappa}:=V_{n}+\kappa W,
$$

where $W \in \mathcal{S}\left(\mathbb{R}^{d}\right)$ is a fixed potential, $V_{n}$ satisfy (5.2), and $|\kappa| \ll 1$ is a small parameter. In [16] it is mentioned that $U_{n, \kappa}$ would be a plausible candidate to disprove the so-called Laptev-Safronov conjecture. This would be achieved if one could show that there is a sequence of eigenvalues $z_{n, \kappa}$ of $-\Delta+U_{n, \kappa}$ such that

$$
\lim _{(n, \kappa) \rightarrow(\infty, 0)} \frac{\left|z_{n, \kappa}\right|^{q-\frac{d}{2}}}{\left\|U_{n, \kappa}\right\|_{q}^{q}}=\infty
$$

for every $q>(d+1) / 2$. For a sequence $z_{n, \kappa} \in \Omega_{\delta}$ (as in the previous example) this is equivalent to

$$
\lim _{(n, \kappa) \rightarrow(\infty, 0)}\left\|U_{n, \kappa}\right\|_{q}^{q}=0 .
$$

It is easy to check that $V_{n}, U_{n, \kappa} \in L^{q}\left(\mathbb{R}^{d}\right)$ for any $q>(d+1) / 2$, with

$$
\left\|V_{n}\right\|_{q}=\mathcal{O}\left(n^{\frac{d+1}{2 q}-1}\right), \quad\left\|U_{n, \kappa}\right\|_{q}=\mathcal{O}\left(n^{\frac{d+1}{2 q}-1}+|\kappa|\right),
$$

and hence (5.5) holds. We now show that a necessary condition for $z_{n, \kappa}$ to be an eigenvalue of $-\Delta+U_{n, \kappa}$ is that

$$
\left|\operatorname{Im} z_{n, \kappa}\right| \leq C \mathrm{e}^{-c n}
$$

for some constants $C, c>0$ and for $n$ large and $|\kappa|$ small enough. In particular, this implies that the bound (3.5) is not saturated for the potentials $U_{n, \kappa}$, and that Corollary 3.3 yields much better bounds in this case. Recall that we assume $\operatorname{Im} z_{n, \kappa}>0$, but the same argument works for $\operatorname{Im} z_{n, \kappa}<0$. The first observation is that in view of (3.5) (or even (3.2)) the condition (5.5) implies

$$
\lim _{(n, \kappa) \rightarrow(\infty, 0)}\left|\operatorname{Im} z_{n, \kappa}\right|=0 .
$$

Hence, we may choose $\delta$ in the definition of $\Omega_{\delta}$ arbitrarily small. Since $U_{n, \kappa} \in$ $L^{q}\left(\mathbb{R}^{d}\right)$ for any $q>(d+1) / 2$ we may fix such a $q$ and a corresponding $\beta_{q}$ as in Corollary 3.3 We first prove (5.7) in the case $\kappa=0$. Since we are only concerned with norms we may substitute $V_{n}$ by the right hand side of (5.2) for the following argument. We may then write the bound (5.1) as

$$
\left\|V_{n}\right\|_{L} \frac{d+1}{2}_{(B(0, R))} \gtrsim 1, \quad \text { where } \quad R:=A \frac{|\ln \epsilon|}{\epsilon}, \quad \epsilon=\operatorname{Im} z_{n, \kappa},
$$


and where $A$ is some sufficiently large constant. We argue by contradiction. Assume that (5.7) failed, i.e. that for any $C, c>0$ there are $n$ and $\kappa$ such that

$$
\frac{\epsilon \mathrm{e}^{c n}}{|\ln \epsilon|} \geq C \text {. }
$$

Note that the logarithmic term can be bounded by an arbitrary power of $\epsilon^{-1}$ and can thus be absorbed into the constants by making $c$ slightly smaller and $C$ slightly larger. A straightforward computation shows that

$$
\left\|V_{n}\right\|_{L}{ }_{\frac{d+1}{2}(B(0, R))} \lesssim \frac{1}{n} \max \left(1, \ln \left(\frac{R}{n}\right)\right) .
$$

If the maximum were in fact 1 , then (5.8) would imply that $n \lesssim 1$, which is absurd; hence we may replace the maximum by $\ln \left(\frac{R}{n}\right)$. Under assumption 5.9 we have

$$
\frac{R}{n} \leq \frac{A}{C} \frac{\mathrm{e}^{c n}}{n} \text {. }
$$

Plugging this into (5.10) yields

$$
\left\|V_{n}\right\|_{L^{\frac{d+1}{2}}(B(0, R))} \lesssim c
$$

for $n$ sufficiently large. Since $c$ was arbitrarily small, this contradicts (5.8). The proof for $\kappa \neq 0$ and $|\kappa|$ sufficiently small is an easy modification of the previous argument. The only difference is that $|\kappa|$ is added to the right hand sides of (5.10) and (5.11).

Example 5.3. The next example is more informal than the previous ones. We consider the "rectangular well"

$$
V:=\alpha \mathbf{1}_{\mathbf{R}}, \quad \mathbf{R}:=[-R, R] \times[-\sqrt{R}, \sqrt{R}] \times \ldots \times[-\sqrt{R}, \sqrt{R}] .
$$

where $\alpha \in \mathbb{C},|\alpha| \ll 1$, and $R \gg 1$ are two parameters. We easily compute

$$
\|V\|_{q} \approx|\alpha| R^{\frac{d+1}{2 q}}, \quad 1 \leq q \leq \infty .
$$

Suppose that $z$ is an eigenvalue of $-\Delta+V$. We omit the dependence of $V$ and $z$ on the parameters $\alpha, r$ in the notation. We denote $\epsilon=\operatorname{Im} z$ and assume that $\epsilon>0$. Since $V$ has support in $\mathbf{R}$, an eigenfunction $u$ (say $L^{2}$ normalized) corresponding to the eigenvalue $z$ decays exponentially outside of $\mathbf{R}$, at a rate $\exp (-\epsilon|x|)$; this follows from inspection of the fundamental solution of $-\Delta-z$. If we multiply the eigenvalue equation $-\Delta u+V u=z u$ by $\mathbf{1}_{\mathbf{R}} \bar{u}$ and integrate by parts, we get informally

$$
\int_{\mathbf{R}}|\nabla u|^{2}+\alpha \int_{\mathbf{R}}|u|^{2}=z \int_{\mathbf{R}}|u|^{2}+\text { (boundary terms). }
$$

To make sense of the boundary terms we may slightly smooth out the rectangle $\mathbf{R}$. In any event, due to the exponential decay of $u$ we have the rough bound

$$
\text { (boundary terms) } \lesssim \exp (-c \epsilon \sqrt{R}) \times \text { (perimeter of } \mathbf{R}) \text {. }
$$

Observe that the worst contribution of the boundary terms comes from those boundary surfaces that involve the long side of the rectangle. On the "good" boundary surfaces one has the better exponential decay $\exp (-c \epsilon R)$. As we have to contend with (5.15), we can neglect boundary terms provided that

$$
\sqrt{R} \geq \frac{C}{\epsilon}(\ln R+|\ln \epsilon|) .
$$


for some sufficiently large constant $C$. It is straightforward to see that if (5.16) is satisfied when $|\ln \epsilon|$ is dropped from the right hand side, then it holds as stated for sufficiently large $R$. We thus assume that

$$
\sqrt{R} / \ln R \geq C / \epsilon,
$$

which also implies that $\sqrt{R} \geq C|\ln \epsilon| / \epsilon$. Taking imaginary parts in (5.14) then yields

$$
(\operatorname{Im} \alpha-\epsilon) \int_{\mathbf{R}}|u|^{2}=\mathcal{O}\left(\epsilon^{2}\right) .
$$

One concludes (still informally) that $\operatorname{Im} \alpha=\epsilon$ up to a small error that goes to zero as $R \rightarrow \infty$ and $\epsilon \rightarrow 0$. Ignoring the $\ln R$ term in (5.17) (since $q>(d+1) / 2$ is fixed we always have an epsilon of room that allows us to replace $\ln R$ by a small power of $R$ ) and plugging this into (5.13) yields that $\|V\|_{q} \gtrsim \epsilon^{1-\frac{d+1}{q}}$; the right hand side only tends to zero if $q>d+1$. The loss of the "2" in the denominator comes from the bad boundary estimate (5.15). Had we only considered the good boundary surfaces we would have the better bound $\|V\|_{q} \gtrsim \epsilon^{1-\frac{d+1}{2 q}}$. To circumvent the problem we simply assume that

$$
R=A \frac{|\ln \epsilon|}{\epsilon}, \quad \alpha:=\mathrm{i} \epsilon .
$$

We also (still) assume that $-\Delta+V$ has an eigenvalue $z$ with $|z| \approx 1, \operatorname{Im} z \approx \epsilon$. From (5.13) and (5.18) it is then easy to see that the bounds (3.5) and (5.8) are saturated up to logarithms.

Example 5.4. We now establish a rigorous version of the last example in one dimension. The claim we are going to prove is the following: Given $\epsilon>0$ sufficiently small, there exists a "complex square well potential" $V=V_{0} \mathbf{1}_{Q}, Q=[-R, R]$, $R \approx|\ln \epsilon| / \epsilon, V_{0} \in \mathbb{C},\left|V_{0}\right| \approx \epsilon$, such that $-\partial^{2}+V$ has eigenvalue $(1+\mathrm{i} \epsilon)^{2}$ and $\|V\|_{1} \approx|\ln \epsilon|$. In particular, the bound (5.8) is saturated up to logarithms.

Proof. Since $V(x)=V(-x)$ the wavefunction $\psi$ must be either even or odd. We consider the even case. Then the Ansatz for $\psi$ is

$$
\psi(x)= \begin{cases}A \mathrm{e}^{-\mathrm{i} z x} & (x \leq-R), \\ B \cos (\mathrm{i} k x) & (-R \leq x \leq R), \\ A \mathrm{e}^{\mathrm{i} z x} & (x \geq R),\end{cases}
$$

where

$$
k^{2}=z^{2}-V_{0}
$$

Continuity of $\psi, \psi^{\prime}$ at $R$ is equivalent to

$$
\operatorname{det}\left(\begin{array}{cc}
\mathrm{e}^{\mathrm{i} z R} & -\cos (k R) \\
\mathrm{i} z \mathrm{e}^{\mathrm{i} z R} & k \sin (k R)
\end{array}\right)=0 \Longleftrightarrow z=\mathrm{i} k \frac{\sin (k R)}{\cos (k R)} .
$$

This can be written as

$$
z=-k \frac{1-\mathrm{e}^{2 \mathrm{i} k R}}{1+\mathrm{e}^{2 \mathrm{i} k R}} .
$$

We make the change of variables

$$
\omega=1+k \in \mathbb{C}, \quad z=1+\mathrm{i} \varepsilon \in \mathbb{C}, \quad 0<\varepsilon<1 / 2 .
$$


For fixed $0<\rho<1$ define a function, depending on the parameters $R>0, \varepsilon>0$,

$$
B(\mathrm{i} \varepsilon, \rho \varepsilon) \ni \omega \mapsto f_{\varepsilon, R}(\omega):=\mathrm{i} \varepsilon+\omega \frac{1-\mathrm{e}^{2 \mathrm{i}(\omega-1) R}}{1+\mathrm{e}^{2 \mathrm{i}(\omega-1) R}} .
$$

Then it can be seen that (5.20) together with the condition $|1+k-\mathrm{i} \varepsilon|<\rho \varepsilon$ is equivalent to $f_{\epsilon, R}(\omega)=0$. We make the assumption that

$$
(1-\rho) \varepsilon R \geq-C-\ln \varepsilon
$$

for some large but fixed constant $0<C<-\ln \varepsilon$, which ensures that

$$
\sup _{\omega \in B(\mathrm{i} \varepsilon, \rho \varepsilon)}\left|\mathrm{e}^{2 \mathrm{i}(\omega-1) R}\right|=\mathcal{O}\left(\varepsilon^{2}\right) .
$$

In particular, we have that

$$
f_{\varepsilon, R}(\omega)=\mathrm{i} \varepsilon+\omega+\mathcal{O}\left(\varepsilon^{2}\right) .
$$

Applying Lemma 2.23 in $[8$ yields that for $\varepsilon$ sufficiently small and $R$ sufficiently large (depending on $\rho$ ) so that (5.22) holds, the function $f_{\epsilon, R}$ has exactly one simple zero in $B(\mathrm{i} \varepsilon, \rho \varepsilon)$, given by

$$
\omega=-\mathrm{i} \varepsilon(1+\mathcal{O}(\varepsilon)) .
$$

This means that (5.20) has a solution $k \in \mathbb{C}$ with $|1+k-\mathrm{i} \varepsilon|<\rho \varepsilon$ and therefore $z^{2}=(1+\mathrm{i} \varepsilon)^{2}$ is an eigenvalue for the Schrödinger operator $-\partial^{2}+V$ with potential $V=V_{0} \mathbf{1}_{[-R, R]}$. Recalling (5.19) we get the estimate

$$
\|V\|_{q}=\left|z^{2}-k^{2}\right| R^{1 / q}=\left|z^{2}-(\omega-1)^{2}\right| R^{1 / q} \leq 2 \varepsilon(2+\rho+\mathcal{O}(\varepsilon)) R^{1 / q} .
$$

Fixing $R$ by requiring equality in (5.22), we get

$$
\|V\|_{q} \approx \varepsilon^{1-1 / q}|\ln \varepsilon|^{1 / q}, \quad 1 \leq q \leq \infty .
$$

Example 5.5. The next example is a simplified version of 2 in $d=3$ dimensions 2 Concretely, we show: Given $\epsilon>0$ sufficiently small, there exists a radial potential supported in $B(0, R), R \approx|\ln \epsilon| / \epsilon$, such that $-\Delta^{2}+V$ has eigenvalue $(1+\mathrm{i} \epsilon)^{2}$ and $\|V\|_{q} \lesssim \epsilon^{1-3 / q}$ for $q>3$ (up to logarithms). The potential in this example is too large to saturate the bound (5.8); in fact,

$$
\|V\|_{L^{2}(B(0, R)} \approx \epsilon^{-\frac{1}{2}} \quad \text { (up to logaraithms). }
$$

Proof. Let $z_{1}, z_{2} \in \mathbb{C}^{+}$(i.e. $\operatorname{Im} z_{j}>0$ ), $A_{1}, A_{2} \in \mathbb{C}$ and $R>0$. All parameters will be determined later. Then set

$$
u(r):= \begin{cases}A_{1} \frac{\sin \left(z_{1} r\right)}{r} & (r \leq R), \\ A_{2} \frac{\mathrm{e}^{\mathrm{i} z} 2^{r}}{r} & (r \geq R) .\end{cases}
$$

In order to have $u \in H^{2}\left(\mathbb{R}^{3}\right)$ it is enough that $u$ and $u^{\prime}$ are continuous at $r=R$. This is the case iff the linear equation

$$
\left(\begin{array}{cc}
\sin \left(z_{1} R\right) & -\mathrm{e}^{\mathrm{i} z_{2} R} \\
z_{1} \cos \left(z_{1} R\right) & -\mathrm{i} z_{2} \mathrm{e}^{\mathrm{i} z_{2} R}
\end{array}\right)\left(\begin{array}{l}
A_{1} \\
A_{2}
\end{array}\right)=\left(\begin{array}{l}
0 \\
0
\end{array}\right)
$$

\footnotetext{
${ }^{2}$ Similarly, the previous example could also be regarded as a simplified one-dimensional version.
} 
has a nontrivial solution $\left(A_{1}, A_{2}\right)^{T}$, hence iff

$$
\operatorname{det}\left(\begin{array}{cc}
\sin \left(z_{1} R\right) & -\mathrm{e}^{\mathrm{i} z_{2} R} \\
z_{1} \cos \left(z_{1} R\right) & -\mathrm{i} z_{2} \mathrm{e}^{\mathrm{i} z_{2} R}
\end{array}\right)=0 \Longleftrightarrow-\mathrm{i} z_{2} \sin \left(z_{1} R\right)+z_{1} \cos \left(z_{1} R\right)=0
$$

In other words, by Euler's formula,

$$
z_{2}=-z_{1} \frac{1+\mathrm{e}^{2 \mathrm{i} z_{1} R}}{1-\mathrm{e}^{2 \mathrm{i} z_{2} R}}
$$

We set $\operatorname{Re} z_{1}=1 / 2$ and write $\operatorname{Im} z_{1}=\varepsilon>0$. Then

$$
\begin{aligned}
\operatorname{Im} z_{2} & =-\varepsilon-\sin (R) \mathrm{e}^{-2 \varepsilon R}+E, \\
|E| & \leq c_{1} \varepsilon^{2}+c_{2} \mathrm{e}^{-4 \varepsilon R} .
\end{aligned}
$$

We set $R=C /(2 \varepsilon)$, with $C_{0} \leq C \leq-\ln ((1+\delta) \varepsilon)$. Here, $\delta>0$ is fixed and $C_{0}>0$ is such that $\mathrm{e}^{-C_{0}}+c_{2} \mathrm{e}^{-2 C_{0}} \geq(1-\delta / 4) \mathrm{e}^{-C_{0}}$. Let $\varepsilon_{0}>0$ be such that $-\varepsilon+c_{1} \varepsilon^{2} \geq-(1+\delta / 4) \varepsilon$ for all $\varepsilon \leq \varepsilon_{0}$. We may also arrange that $\sin (R)=-1$.

Then

$$
\begin{aligned}
\operatorname{Im} z_{2} & \geq-(1+\delta / 4) \varepsilon+(1-\delta / 4) \mathrm{e}^{-C} \\
& \geq-(1+\delta / 4)+(1-\delta / 4)(1+\delta) \varepsilon=\left(\delta / 2+\mathcal{O}\left(\delta^{2}\right)\right) \varepsilon .
\end{aligned}
$$

Hence, there is $\delta_{0}>0$ such that for all $\delta \leq \delta_{0}$

$$
\operatorname{Im} z_{2} \geq \frac{\delta}{4} \varepsilon
$$

Then $u$ satisfies

$$
\left|\left(\Delta+z_{2}^{2}\right) u(r)\right| \lesssim \mathrm{e}^{-C} \mathbf{1}\{r \leq C /(2 \varepsilon)\}|u(r)| .
$$

Hence, if $V$ is defined by the equation $\left(\Delta+z_{2}^{2}-V(r)\right) u(r)=0$, we have

$$
\|V\|_{q} \lesssim \mathrm{e}^{-C}(C / \varepsilon)^{3 / q}
$$

The minimum is achieved for $C=-\ln ((1+\delta) \varepsilon)$, namely

$$
\|V\|_{q} \lesssim \varepsilon^{1-3 / q} \quad \text { up to } \ln (\varepsilon) .
$$

This tends to 0 as $\varepsilon \rightarrow 0$, provided $q>d$. Note that since $\mathrm{e}^{\mathrm{i} z_{2} r} / r$ is a fundamental solution to $-\Delta-z_{2}^{2}$, the support of $V$ is contained in $B(0, R)$.

Example 5.6. Here we prove that the result of Proposition 4.1] is sharp. We could take the rectangular well potential as in Example 5.3. but for the sake of variety we consider a Gaussian potential $G(t)=\exp \left(-t^{2} / 2\right)$. By slight abuse of notation we understand that $\epsilon>0$ is a sequence tending to zero. We suppress the dependence of $V, \psi, g$ in Proposition 4.1 on the index $n$ of this sequence. We start with the quasimode

$$
\psi(x):=N^{-1 / 2} \mathrm{e}^{\mathrm{i} x_{1}} G(|y|)_{y=\left(\epsilon x_{1}, \sqrt{\epsilon} x^{\prime}\right)},
$$

where $x=\left(x_{1}, x^{\prime}\right) \in \mathbb{R} \times \mathbb{R}^{d-1}$ and $N=\epsilon^{-\frac{d+1}{2}}$ is a normalization factor. We compute

$$
(-\Delta-1-\mathrm{i} \epsilon) \psi(x)=\left(\epsilon\left(d-1-\left|y^{\prime}\right|^{2}+2 \mathrm{i} y_{1}-\mathrm{i}\right)+\epsilon^{2}\left(1-y_{1}^{2}\right)\right) \psi(x),
$$

where, as before, $y=\left(\epsilon x_{1}, \sqrt{\epsilon} x^{\prime}\right)$. Hence, if $V(x)=\epsilon \chi(y)_{y=\left(\epsilon x_{1}, \sqrt{\epsilon} x^{\prime}\right)}$, where $\chi$ is an arbitrary Schwartz function, say with $\chi(0)=1$, we get

$$
\|g\|_{2}:=\|(-\Delta-1-\mathrm{i} \epsilon+V) \psi\|_{2} \lesssim \epsilon, \quad\|V\|_{q} \approx \epsilon^{1-\frac{d+1}{2 q}}, \quad\left\|V^{\frac{1}{2}} \psi\right\|_{2} \approx 1 .
$$


This implies that condition (4.2) holds with a good margin and that (4.3) is sharp.

Example 5.7. We show that the quasimode bound $\|g\|_{2} \lesssim \epsilon$ of the previous example may be improved (by changing the potential) to an exponentially small error. For this we set

$$
V(x):=\epsilon V_{1}(x)+\epsilon^{2} V_{2}(x)
$$

where

$$
\begin{aligned}
& V_{1}(x):=-\left(d-1-\left|y^{\prime}\right|^{2}+2 \mathrm{i} y_{1}-\mathrm{i}\right) \mathbf{1}_{|y| \leq M}, \\
& V_{2}(x):=-\left(1-y_{1}^{2}\right) \mathbf{1}_{|y| \leq M},
\end{aligned}
$$

and $M \gg 1$ will be chosen later. Then (5.26) yields

$$
\|g\|_{2}:=\|(-\Delta-1-\mathrm{i} \epsilon+V) \psi\|_{2} \lesssim \epsilon \exp \left(-\frac{1}{4} M^{2}\right) .
$$

For fixed $(d+1) / 2<q<\infty$ there exists $\delta \in(0,1)$ such that $q=(d+1) /(2(1-\delta))$. Then

$$
\|V\|_{q} \lesssim \epsilon^{\delta} M^{2+\frac{d}{q}} .
$$

We now choose $M=\epsilon^{-\frac{\delta}{2(2+d / q)}}$, leading to

$$
\|g\|_{2} \lesssim \epsilon \exp \left(-\frac{1}{4} \epsilon^{-\frac{\delta}{(2+d / q)}}\right), \quad\|V\|_{q} \lesssim \epsilon^{\frac{\delta}{2}} .
$$

Acknowledgements: The author gratefully acknowledges the hospitality of the Institut Mittag-Leffler and the invitation to the thematic program Spectral Methods in Mathematical Physics. The present article was written during the authors stay. The idea to use Lemma 4.3 and the proof thereof is attributed to Chris Sogge, to whom the author is thankful.

\section{REFERENCES}

[1] A. A. Abramov, A. Aslanyan, and E. B. Davies. Bounds on complex eigenvalues and resonances. J. Phys. A, 34(1):57-72, 2001.

[2] S. Bögli. Schrödinger operator with non-zero accumulation points of complex eigenvalues. Comm. Math. Phys., 352(2):629-639, 2017.

[3] J.-C. Cuenin. Eigenvalue bounds for Dirac and fractional Schrödinger operators with complex potentials. J. Funct. Anal., 272(7):2987-3018, 2017.

[4] J.-C. Cuenin. Embedded eigenvalues for generalized Schrödinger operators. arXiv:1709.06989 page 18 pages, 2017.

[5] E. B. Davies and J. Nath. Schrödinger operators with slowly decaying potentials. J. Comput. Appl. Math., 148(1):1-28, 2002. On the occasion of the 65th birthday of Professor Michael Eastham.

[6] M. Demuth, M. Hansmann, and G. Katriel. On the discrete spectrum of non-selfadjoint operators. J. Funct. Anal., 257(9):2742-2759, 2009.

[7] M. Demuth, M. Hansmann, and G. Katriel. Eigenvalues of non-selfadjoint operators: a comparison of two approaches. In Mathematical physics, spectral theory and stochastic analysis, volume 232 of Oper. Theory Adv. Appl., pages 107-163. Birkhäuser/Springer Basel AG, Basel, 2013.

[8] S. Dyatlov and M. Zworski. Mathematical theory of scattering resonances. http://math.mit.edu/ dyatlov/res/res_final.pdf 2019.

[9] L. Fanelli, D. Krejčiř́k, and L. Vega. Absence of eigenvalues of two-dimensional magnetic Schrödinger operators. J. Funct. Anal., 275(9):2453-2472, 2018. 
[10] L. Fanelli, D. Krejčiřík, and L. Vega. Spectral stability of Schrödinger operators with subordinated complex potentials. J. Spectr. Theory, 8(2):575-604, 2018.

[11] R. L. Frank. Eigenvalue bounds for Schrödinger operators with complex potentials. Bull. Lond. Math. Soc., 43(4):745-750, 2011.

[12] R. L. Frank. Eigenvalue bounds for Schrödinger operators with complex potentials. III. Trans. Amer. Math. Soc., 370(1):219-240, 2018.

[13] R. L. Frank, A. Laptev, E. H. Lieb, and R. Seiringer. Lieb-Thirring inequalities for Schrödinger operators with complex-valued potentials. Lett. Math. Phys., 77(3):309-316, 2006.

[14] R. L. Frank, A. Laptev, and O. Safronov. On the number of eigenvalues of Schr'odinger operators with complex potentials. ArXiv e-prints, Jan. 2016.

[15] R. L. Frank and J. Sabin. Restriction theorems for orthonormal functions, Strichartz inequalities, and uniform Sobolev estimates. Amer. J. Math., 139(6):1649-1691, 2017.

[16] R. L. Frank and B. Simon. Eigenvalue bounds for Schrödinger operators with complex potentials. II. J. Spectr. Theory, 7(3):633-658, 2017.

[17] C. Guillarmou, A. Hassell, and K. Krupchyk. Eigenvalue bounds for non-self-adjoint Schrödinger operators with non-trapping metrics. ArXiv e-prints, Sept. 2017.

[18] D. Hundertmark, E. H. Lieb, and L. E. Thomas. A sharp bound for an eigenvalue moment of the one-dimensional Schrödinger operator. Adv. Theor. Math. Phys., 2(4):719-731, 1998.

[19] A. D. Ionescu and D. Jerison. On the absence of positive eigenvalues of Schrödinger operators with rough potentials. Geom. Funct. Anal., 13(5):1029-1081, 2003.

[20] C. E. Kenig, A. Ruiz, and C. D. Sogge. Uniform Sobolev inequalities and unique continuation for second order constant coefficient differential operators. Duke Math. J., 55(2):329-347, 1987.

[21] A. Laptev and O. Safronov. Eigenvalue estimates for Schrödinger operators with complex potentials. Comm. Math. Phys., 292(1):29-54, 2009.

[22] Y. Lee and I. Seo. A note on eigenvalue bounds for Schrödinger operators. J. Math. Anal. Appl., 470(1):340-347, 2019.

[23] E. H. Lieb and W. Thirring. Inequalities for the moments of the eigenvalues of the schrodinger hamiltonian and their relation to sobolev inequalities. Studies in Mathematical Physics. Princeton University Press, 1976.

[24] H. Mizutani. Eigenvalue bounds for non-self-adjoint Schr \"odinger operators with the inversesquare potential. ArXiv e-prints, July 2016.

[25] E. M. Stein and G. Weiss. Introduction to Fourier analysis on Euclidean spaces. Princeton University Press, Princeton, N.J., 1971. Princeton Mathematical Series, No. 32.

Mathematisches Institut, Ludwig-Maximilians-Universität München, 80333 Munich, GERMANY

E-mail address: cuenin@math.lmu.de 